\title{
Visuelle Narration. Zum journalistischen Storytelling aus der Sicht der Medienlinguistik
}

Magdalena Makowska (Olsztyn)

\begin{abstract}
From among many narrative forms used in the media, storytelling starts to occupy a more and more important place. Its essence constitutes the telling of a story which must be well understandable as well as easy to remember and retell. Storytelling is one of the best methods of awakening interest and focusing the recipient's attention. Owing to the adequate manner of telling a story as well as its appropriate structuring particular needs are evoked in recipients. The mystery of effective usage of storytelling consists in the fact that it is essential to appeal to emotions and to tell a given story that will carry some message with it in a consistent way and will also inspire one to engage in communication activities. That makes storytelling an interesting subject of media-linguistic research whose effects - as illustrated by the history of a plane crash of the Germanwings lines told in the form of an online reportage - will be presented in this article.
\end{abstract}

"Nicht der Print oder Online ist wichtig, sondern die Geschichte, auf welcher Plattform auch immer sie gelesen wird.“ (Alan Rusbringer, Chefredakteur des Guardian, zitiert nach Becker 2010)

\section{$1 \quad$ Einleitung}

Neben vielen anderen Wenden, die moderne Kommunikation prägen, spielt die Wende, zu der es im Bereich der Narrativität kommt, eine immer wichtigere Rolle. Zwar begleitet das Geschichtenerzählen die Menschheit seit jeher, aber das Potenzial der Narrativität ist trotzdem immer noch nicht ausgeschöpft. In der modernen Zeit neigen Menschen sogar dazu, auch das, was seiner Natur nach nicht-narrativ zu sein scheint, doch zu narrativisieren, d. h. als Geschichte zu gestalten und zu erzählen (vgl. Handler 2006). Die Autoren greifen auf Narrationen zurück, um komplexe Ereignisse zu vergegenwärtigen, zu erfassen und zu interpretieren. Narrationen ermöglichen den Menschen, aus der Fülle der zunächst chaotischen Wahrnehmungen das herauszufiltern, was bedeutsam sein kann. Nicolas Pethes (2008) zufolge ist 
[...] die Ereignisvielfalt des Lebens überhaupt nur durch narrative Operationen zu bewältigen [...]. Die Selektion solcher Ereignisse, die sich als zusammenhängende Entwicklung wahrnehmen lassen, die Angabe kausaler Zusammenhänge zwischen ihren einzelnen Etappen, die wieder erkennbare Charakterisierung der beteiligten Personen sowie die Auswahl einer Perspektive auf die Vorgänge, die diese nachvollziehbar werden lässt, sind allesamt Techniken des Erzählens, die die Wahrnehmbarkeit der Vergangenheit allererst ermöglichen und auf diese Weise bereits das gegenwärtige Erleben selbst strukturieren.

(Pethes 2008: 124)

Das multimodal realisierte Geschichtenerzählen wird heutzutage sowohl für ein Mittel der Wissensvermittlung als auch immer häufiger für ein Medium der Überzeugung gehalten, das u. a. in Publizistik oder Werbung nützlich sein kann. Die Narrativität wird somit zu einer transdisziplinären Schlüsselkategorie, die es möglich macht, die Brücken zwischen unterschiedlichen Lebensbereichen zu schlagen. Auch im Journalismus erlebt die Kunst des Erzählens eine Renaissance: Infolge des technologischen Fortschrittes können Medienformate je nach ihrer Verwendung funktional und gestalterisch variiert werden (vgl. Perrin 2010: 145). Peter Schumacher weist darauf hin, dass z. B. „das Internet mit seinen Eigenschaften der Hypertextualität, Multimodalität und Interaktivität eine Plattform für neuartige journalistische Darstellungsformen bietet" (Schumacher 2012a: 311). Während sich z. B. eine prototypische Reportage, die man mit dem Printmedium Zeitung assoziiert, diesem Medium (= schriftsprachlicher Text und statische Bilder) anpasst, kann eine Online-Reportage über ganz andere technische Möglichkeiten verfügen und aus anderen medialen Lösungen schöpfen. Die AutorInnen von OnlineReportagen nutzen die Ressourcen einer Medienorganisation, die ihnen computerbasierte Technik zur Verfügung stellt, um u. a. die Narrativität attraktiver zu machen. Somit wird die Geschichte gleichzeitig auf mehreren, multimodal realisierten Ebenen erzählt und schöpft aus dem semantischen, funktionalen und formalen Potenzial dessen, was sie diesen Ebenen und allen ihnen zugrundeliegenden sprachlichen, bildlichen, grafischen oder auch auditiven Ressourcen zu verdanken hat.

Die im Zentrum des vorliegenden Beitrags stehende Frage nach Möglichkeiten und Strategien multimodalen Geschichtenerzählens wird am Beispiel einer Online-Reportage thematisiert. Den theoretischen Hintergrund dieser Analyse bilden medienlinguistische Überlegungen zur Multimodalität und Rezeption von multimodalen Darstellungsformen sowie zu ihrem Platz im digitalen Journalismus (vgl. Bucher/Schumacher 2012, Baechler/Eckkrammer u. a. 2016). Das Datenkorpus bildet die Online-Reportage Absturz von Flug 4U9525, die der Westdeutsche Rundfunk unter der Adresse http://reportage.wdr.de/absturz-germanwings publiziert hat und die dem Absturz eines Germanwings-Flugzeuges gewidmet wird. Der vorliegende Beitrag ist anwendungsorientiert und setzt sich zum Ziel, Vorschläge dafür zu thematisieren, wie sich Rezeptionsprobleme durch eine nutzergerechte Gestaltung vermeiden lassen und wie das Storytelling zur Verbesserung der Qualität im digitalen Journalismus beitragen kann. Am Beispiel der analysierten Online-Reportage werden u. a. Fragen danach gestellt, was die Narrativität in einem solchen online realisierten Text ausmacht, $d$. h. welcher strukturellen, kommunikativen und dramaturgischen Ausrichtung die Sequenzierung der Website unterliegt und welche sprachlichen und gestalterischen Mittel die analysierte Online-Reportage als kohäsives und kohärentes Gesamtkommunikat aufweist. 


\section{Multimodalität und multimodale Darstellungsformen als Anzeichen des Medien- wandels}

Digital technologies are of particular interest to multimodality because their design and situated use tends to make a wide range of modes available, often in new inter-semiotic relationships with one another, and in ways that unsettle and remake genres, as well as in ways that reshape practices and interaction.

(Jewitt 2014: 452)

Heutige Kommunikation steht unter dem Einfluss eines hochdynamischen Medienwandels. Als Anzeichen dieses Wandels gilt die rasante Entwicklung von Technologien, die einen wichtigen Beitrag nicht nur zur Beschleunigung, sondern auch zur qualitativen Verbesserung der Kommunikation leisten kann. Das betrifft u. a. den Journalismus, der immer häufiger verschiedene Distributionskanäle zum Zweck der Informationsvermittlung nutzt und diese Informationen gerne narrativisiert, d. h. als Geschichten präsentiert. Außer geschriebener Sprache, der im Journalismus immer noch eine zentrale Rolle zukommt, wird dabei gleichzeitig auf eine Palette von typographischen Hervorhebungen, aussagekräftigen Bildern sowie (Audio-) und Videosequenzen zurückgegriffen, die im Dienste der Narration und Dramaturgie eingesetzt werden. Technologische Neuigkeiten, die in die journalistische Kommunikationspraxis aufgenommen werden, ermöglichen eine immer größere Vielfalt von Kommunikationsangeboten, die dazu tendieren, Informationen multimodal zu gestalten und multimedial zu vermitteln. Somit werden Multimodalität und Multimedialität zu einem der wichtigsten Merkmale der im Journalismus genutzten visuellen Narration, die im Zentrum des vorliegenden Beitrags steht und am Beispiel einer Online-Reportage thematisiert wird.

\subsection{Universalie: Multimodalität}

Multimodale Orchestrierung. So beschreiben Hans-Jürgen Bucher und Peter Schumacher die Art und Weise der Gestaltung von Medienangeboten, die ,[...] in ihrer Komplexität weit über Texte und Text-Bild-Kombinationen hinausgehen“ (Bucher/Schumacher 2012: 10). Das lässt sich gut am Beispiel von Zeitungen beobachten, die Schröder (2010) zufolge von einem Textmedium zu einem Medium der visuellen Kommunikation geworden sind. Die Multimodalität solcher Medienangebote kommt dadurch zum Ausdruck, dass sie Text, statische und bewegte Bilder, Grafik oder Design zusammenbringen. Dadurch, dass sie als Orchestrierungen bezeichnet werden, wird ihre semantische, strukturelle und funktionale Komplexität hervorgehoben. Die Aufgabe von so konstruierten Medienangeboten besteht aber nicht nur darin, etwas attraktiv zu kommunizieren. Den technologischen Lösungen ist u. a. die Verbesserung der Effektivität im Bereich der medialen Vermittlung zu verdanken. Außerdem macht die Technik auch das möglich, dass die Rezipienten zu einer Art der Interaktion animiert werden können, indem sie hypertextuell realisierte Kommunikate je nach ihren individuellen Bedürfnissen und Interessen wahrnehmen und nutzen können. In diesem Sinne „,[prägen] Medientechnologien und ihre Kommunikationsformen den Gebrauch von Sprache und [beeinflussen] dadurch soziale Bezüge, Teilhabe und ideologische Machtgefüge“, so Stöckl (2016b: 22). 
Die neuen, internetbasierten Kommunikationsumgebungen begünstigen die Zunahme und Intensivierung von Multimodalität. Angesichts des dynamischen Medienwandels werden die RezipientInnen, darunter auch die LeserInnen von journalistischen Beiträgen, ständig mit neuartigen multimodalen Botschaften konfrontiert und müssen in der Lage sein, mit ihnen erfolgreich umzugehen. Es lässt sich beobachten, dass verschiedene Textsorten prädigitaler Medienwelten in die technischen Plattformen und Interaktionsumgebungen der neuen Medien migrieren und dabei transformiert werden. So unterscheidet sich eine Online-Reportage, die aus dem Potenzial der digitalen Umgebung und deren Lösungen schöpft, von einer prototypischen Reportage, die man mit einer Printzeitung assoziiert und der ausschließlich statische Ressourcen zur Verfügung stehen. Die Online-Reportage wird oft nicht nur multimodal, sondern auch multimedial oder sogar interaktiv realisiert, was sie von der prototypischen Textsorte Reportage grundsätzlich unterscheidet und was einen wesentlichen Einfluss auf den Rezeptionsprozess nimmt.

Roman Opiłowski verweist darauf, dass „,die kommunikativ-funktionale Interaktion von sprachlichen, bildlichen und auditiven Zeichen Texte während des Rezeptionsprozesses [ausmacht]“ (Opiłowski 2015: 12). Dem Autor zufolge ,,[werden] die Informationen nun in Texten über unterschiedliche semiotische Kodes vermittelt, die die Komplexität und Menge der kommunizierten Inhalte für den Rezeptionsprozess reduzieren, in einzelne semiotische Textfelder verteilen und die Rezeption als solche vereinfachen" (ebd.: 11). Einerseits werden moderne Kommunikate mit Bildern, Graphiken und sogar Tönen angereichert, andererseits müssen sie aber auch immer mehr den Regeln der semiotischen Ökonomie folgen, wenn sie zu unterschiedlichen Ressourcen greifen, um den Rezipienten Informationen am besten nahe zu bringen. Sonst besteht die Gefahr, dass die Vielfalt von gebrauchten Ressourcen den Nutzer leicht überfordern kann. Die AutorInnen multimodaler Medienangebote sollten sich somit zum Ziel setzen, bestimmte Inhalte unterschiedlicher semiotischer und medialer Herkunft erstens effektiv zu vermitteln und zweitens attraktiv darzustellen. Da die dabei gebrauchten Ressourcen über unterschiedliche Leistungskraft verfügen und sich wechselseitig semantisieren können, kann ihr gut durchdachter Gebrauch dazu führen, dass ein Optimum an Informationsleistung erzielt und die Rezeption erleichtert wird.

Medienrezeption kann nicht auf die Erfassung von Inhalten oder Informationen über Schrifttexte reduziert werden, sondern ist als Interpretationsprozess zu sehen, in dem die vielfältigen Symbolsysteme zu einem kohärenten Gesamtsinn integriert werden müssen.

(Bucher/Schumacher 2012: 10)

Hans-Jürgen Bucher (2012: 18) zufolge sollte die Medienrezeption als „eine Ausdifferenzierung des komplexen Zusammenhangs von Medien und ihren individuellen und gesellschaftlichen Folgen“ verstanden werden. Es steht außer Zweifel, dass der Prozess der Rezeption sowohl durch äußere als auch innere Faktoren beeinflusst wird. Unter äußeren Faktoren werden u. a. situative und soziale Einflüsse verstanden, die bestimmten Gruppen gemeinsam sein können (z. B. ein trauriges Ereignis, das die ganze Gesellschaft miterlebt und das zum Thema einer medialen Debatte wird). Als äußerer Faktor ist aber auch das zu klassifizieren, ,in welcher Mediengattung, in welchem Typus einer Mediengattung, in welcher Aufmachungsform, in welcher Darstellungsform, in welcher Textform und in welcher sprachlichen und bildlichen Gestaltung die Inhalte und Informationen präsentiert werden“ (ebd.: 19): z. B. Berichte im Rundfunk - auditiv, in der Zeitung - visuell, d. h. sprachlich und bildlich, im Internet - auditiv und 
visuell, dazu oft noch interaktiv. Im Gegensatz dazu haben innere Faktoren einen stark individuellen Charakter und unterscheiden sich je nach dem Rezipienten, seinem Weltwissen, seiner Erfahrung, usw.

Inhalte, die via Internet präsentiert werden, können aus ganz unterschiedlichen Online-Angeboten zusammengesetzt sein. „Auch wenn nach wie vor im klassischen Onlinejournalismus Darstellungen dominieren, die allein auf Schrifttext und Fotos basieren, haben sich doch in den letzten Jahren eine Reihe von neuen, onlinespezifischen Formen entwickelt“ (Schumacher 2012a: 311). Dazu gehören z. B. Live-Ticker, Fotogalerien oder interaktive Angebote (vgl. Meier 2016: 413). Ihr Einsatz kann dazu führen, dass die Website einer hohen inhaltlichen und verknüpfungstechnischen Dynamik unterliegt. Sprachliche und bildliche Ressourcen werden hypertextuell so verknüpft, dass jeder Rezipient seinen eigenen Weg durch die multimodale und multimediale Botschaft gehen kann. Ernest W. B. Hess-Lüttich vertritt die Meinung, dass der Hypertext die Freiheit des Nutzers in diesem Sinne erweitert, dass dieser vom passiv rezipierenden Leser zum aktiv in den Textprozess eingreifenden Co-Autor wird (vgl. Hess-Lüttich 1997: 134). Ewa Żebrowska (2013: 147) weist darauf hin, dass der Nutzer den Weg der Rezeption zwar frei gestaltet, aber trotzdem durch eine vom Autor konstruierte Strukturierung beschränkt ist und dieser mehr oder weniger bewusst folgt. Schmitz zufolge schafft der Nutzer dabei doch „seinen eigenen semiotischen Raum“ (Schmitz 2001: 159), indem er den Sinn aus dem vorgegebenen Material konstruiert und sich individuell einen Weg durch die Hypertextstruktur bahnt. Somit wird er zu einem Navigator: Zwar entscheidet er allein, welchen Weg er im Prozess der Rezeption gehen will, aber trotzdem bewegt er sich immer noch in einem physischen Raum, dessen Grenzen vom Autor bestimmt werden. Die NutzerInnen übernehmen somit die Verantwortung für die Gestaltung des Kommunikats, das sie aus dem vom Autor zur Verfügung gestellten Material konstruieren. Behilflich sind dabei alle Mittel, die die Usability von Hypertexten verbessern können.

\subsection{Zur Usability von multimodalen Darstellungsformen im digitalen Journalismus}

Multimodale Darstellungsformen sind für Peter Schumacher diejenigen Kommunikate, zu deren zentralen Merkmalen die Multimodalität und Interaktivität gehören und die sich diesen Eigenschaften entsprechend kurz als IMD bezeichnen lassen (vgl. Schumacher 2012a: 311). Ihr Vorteil besteht darin, dass sie verschiedene Verflechtungen von multimodalen Ressourcen möglich machen und Rezipienten aktivieren. Um die Gefahr zu minimalisieren, dass zu viele Informationen in zu vielen verschiedenen Modi angeboten werden, was den Nutzer überfordern kann, ist es wichtig, nach solchen Strategien im Prozess der Produktion und des Gebrauchs von IMD zu suchen, die es erlauben, diese Botschaften sowohl inhaltlich als auch strukturell und funktional optimal zu gestalten. Peter Schumacher weist darauf hin, dass IMD ein Multitasking des Nutzers erfordern: „Er muss

- die Navigationsoptionen finden, verstehen und nutzen

- einen Überblick über jede Seite bekommen und die aktuelle Position innerhalb des Angebots bestimmen können

- Informationen in verschiedenen Modi wie Text, Grafik, Foto und Animation interpretieren und zueinander in eine sinnvolle, kohärente Beziehung setzen“"

(ebd.: 313) 
Im Falle von medial vermittelten und hypertextuell konstruierten IMD hat der Nutzer nicht das ganze Angebot vor Augen. Seine Aufgabe besteht darin, zwischen unmittelbar nacheinander präsentierten und rezipierten Modulen Kohärenz aufzubauen. Peter Schumacher zufolge bedeutet die sinnvolle Modularisierung, ,die Inhalte so zu portionieren und zu strukturieren, dass der Nutzer sie ohne Überforderung rezipieren kann“ (ebd.: 314). Um die Kohärenz zwischen den einzelnen Elementen von IMD zu erreichen, kann man von verschiedenen Mitteln der Oberflächenstruktur Gebrauch machen, die dem Rezipienten eine bessere Orientierung gewährleisten. Je nach der ausgeübten Funktion unterscheidet Stefan Meier folgende Arten von Zeichen in online-diskursiven Kontexten (vgl. Meier 2016: 415):

- repräsentationale Zeichen, die dazu dienen, Informationen und Inhalte zu übermitteln (z. B. Nachrichtentexte, Teaser, Fotos)

- identifikatorische Zeichen, die die Markierung des Autors bzw. des Betreibers der Webseite ermöglichen (z. B. Logos, Profilbilder)

- Ordnungszeichen, die verschiedene Möglichkeiten zur Oberflächenstrukturierung (z. B. Linien, Flächen) und Navigationsstruktur (z. B. Linkpfade) signalisieren

- operationale Zeichen als Ressourcen zur Markierung von Navigationsinstrumenten (z. B. kleine Pfeile, Buttons)

- partizipatorische Zeichen, mit Hilfe deren interaktive Aufgaben signalisiert werden

- $\quad$ appellative Zeichen, die als Einladung dazu dienen, weitere Produkte und Dienstleistungen zu besuchen (z. B. Banner, Anzeigen).

Die strukturelle Modularisierung ermöglicht das „scannende Lesen“ (ebd.: 416): Obwohl die ganze Sehfläche (Schmitz 2011) wahrgenommen wird, hängt vom Rezipienten ab, welchen Modulen bzw. Subflächen er wie viel Zeit und Aufmerksamkeit schenken möchte. Im Falle von Websites unterscheidet Chiew (2004: 135-137) zwischen drei Typen von modularen Struktureinheiten, die im Dienste der Usability stehen:

- Item, d. h. ein multimodales Grundelement auf der Einzelseite, das als ein einer kommunikativen Funktion zugeordnetes und durch Screendesign hergestelltes Zeichenensemble fungiert

- Lexia, d. h. die einzelne Stelle einer Website, die größer als die Sichtfläche sein kann, deshalb werden dem Rezipienten solche Mittel wie das Scrollen zur Verfügung gestellt

- Cluster, das die Form einer hypertextuell mit der Website vernetzten Bedeutungseinheit hat (z. B. eine Startseite, wo die einzelnen Teilthemen präsentiert werden).

Der digitale Journalismus schöpft aus der Kraft der IMD. Volker Lilienthal verweist darauf, dass technische Möglichkeiten, die die Digitalisierung mit sich bringt, dazu beitragen können, dass der digitale Journalismus , der bessere, der ambitioniertere Journalismus des neuen Zeitalters werden“ könnte (Lilienthal 2011: 50). Unabhängig davon, von welchen Ressourcen und in welchen Kombinationen Gebrauch gemacht wird, ist und bleibt aber das Wichtigste im digitalen Journalismus die Fähigkeit, spannende Geschichten zu recherchieren und zu erzählen. Deshalb werden im Folgenden die zentralen Probleme des erfolgreichen Geschichtenerzählens exemplarisch dargestellt und erläutert. Der besondere Fokus wird daraufgelegt, wie die einzelnen Modi der IMD um die Aufmerksamkeit des Nutzers konkurrieren und das Geschichtenerzählen fördern. 


\section{$3 \quad$ Online-Reportage als Beispiel für digitales Storytelling}

Für Simon Sturm ist eine gut erzählte Geschichte der beste Transportweg für Informationen im heutigen Journalismus (vgl. Sturm 2013: 30). Dem Autor zufolge ist es auch legitim, Storytelling für ,eine uralte, tief in der Gesellschaft verwurzelte Form der narrativen Wissensvermittlung“ (ebd.) zu halten. Eine medial vermittelte Geschichte, die den Regeln des Storytellings folgt, ist dadurch gekennzeichnet, dass anschauliche Texte, eindrucksvolle Videos, aussagekräftige Bilder und authentische Audioaufnahmen miteinander so verknüpft werden, dass eine Art der Dramaturgie gebildet wird, die bei dem Rezipienten Emotionen weckt und Assoziationen evoziert. Mittels sprachlicher, bildlicher und akustischer Elemente kann in Form eines Hypertextes eine so dichte Atmosphäre geschaffen werden, dass der Nutzer in die Geschichte hineingezogen wird und diese miterlebt. Das ist deshalb möglich, weil das Potenzial des Storytellings vor allem in der Bedeutung gründet, die Erzähltexte für das Erkennen und Verstehen von Emotionen haben. Menschliche Emotionen sind abhängig von der Geschichte, in die sie eingebettet werden. Deshalb ist die Fähigkeit, der Situation angemessene Erzählungen über die Erlebnisse von anderen anzufertigen, von zentraler Bedeutung für zwischenmenschliche Kommunikation. Ob der Rezipient mitfühlend auf die Emotion des Erzählers reagiert und selbst eine ähnliche Emotion empfindet, hängt u. a. davon ab, wie eine Geschichte erzählt wird.

\subsection{Der Hintergrund der analysierten Online-Reportage und deren mediale Realisie- rung}

Die Geschichte des Germanwings-Absturzes gilt als gutes Material für digitales Storytelling. Zur Katastrophe des Germanwings-Flugzeuges kam es am 24. März 2015 auf dem Gebiet der Gemeinde Prads-Haute-Bléone in Frankreich. Am Bord gab es 150 Insassen (darunter 16 Schüler), die gar keine Chance hatte, zu überleben. Im Ermittlungsverfahren wurde festgestellt, dass der unter Depression leidende Copilot das Flugzeug willentlich zum Absturz gebracht hat, indem er die Zeit nutzte, als der Kapitän außerhalb des Cockpits war.

Die Geschichte des Germanwings-Absturzes verfügt über innere, natürliche Dramaturgie (Verlauf des Fluges) und weckt so unterschiedliche Emotionen wie Trauer um die Opfer und Hass gegen den Copiloten, der sich für den Selbstmord entschieden und dabei auch den Tod von Passagieren verursacht hat. Weil die Katastrophe weltweit kommentiert wurde, war das dokumentierende Material sehr umfangreich und es gab viele aussagekräftige Bilder und authentische Audio- oder Videoaufnahmen, die Journalisten nutzen konnten, um diese dramatische Geschichte spannend zu erzählen und wahrheitsgemäß zu dokumentieren. Die Methode des journalistischen Storytellings, die aus verschiedenen digitalen Möglichkeiten schöpft, war deshalb für diesen Fall optimal geeignet und galt auch als Basis für die Online-Reportage Absturz von Flug 4U9525, die der Westdeutsche Rundfunk unter der Adresse http://reportage.wdr.de/absturz-germanwings zur Verfügung gestellt hat.

Aus medienlinguistischer Perspektive ist die analysierte Online-Reportage eine multimodale Darstellungsform (IMD), die sich als Transportweg des Erzählten zum Ziel setzt, die Geschichte auf die bestmögliche Weise zum Empfänger zu bringen. Sie lässt sich mit einem ähnlichen Instrumentarium beschreiben, das auch im Falle von Websites in Betracht gezogen wird. 
Je nachdem, was für ein Handlungstyp für eine Website dominant ist, lassen sich Meier zufolge nachrichtenorientierte, imageorientierte, kampagnenorientierte und plattform- bzw. portalorientierte Websites unterscheiden (vgl. Meier 2002: 302-304). Folgt man dieser Klassifikation, kommt man zur Schlussfolgerung, dass sich die im vorliegenden Beitrag analysierte OnlineReportage mit der von Meier präsentierten Klassifikation nicht direkt beschreiben lässt. OnlineReportagen erzählen Geschichten und haben keine Werbefunktion. Anders als nachrichtenorientierte Websites brauchen sie auch nicht ständig aktualisiert zu werden, weil die erzählte Geschichte über ihre eigene, innere Dramaturgie (Anfang, Mitte, Ende) verfügt und in diesem inhaltlichen Sinne eine abgeschlossene Einheit ist. Deshalb scheint es begründet zu sein, die von Meier vorgeschlagene Klassifikation von Websites um einen Typus zu erweitern. Aufgrund der dominierenden Funktion wird der fünfte Typus als erzählende bzw. dokumentierende Websites bezeichnet. Websites, die diesen Typus repräsentieren, fokussieren Ereignisse, die aus gesellschaftlicher Sicht relevant sein können und/oder über potenziellen historischen Wert verfügen. Die historische Perspektive bedingt, dass diese Websites entstehen, um eine Geschichte zu erzählen und zu popularisieren, aber sie tun das ohne kommerzielles Ziel. Anders als im Falle von kampagnenorientierten Websites wird dabei zu keinen Aktionen aufgerufen, sondern es wird etwas erzählt, was schon passiert ist. Erzählende Websites werden somit zu einem digital realisierten Archiv. Den Journalisten, die sich mit Online-Reportagen beschäftigen, wird somit „ein ganzes Panorama neuer Möglichkeiten [eröffnet - MM], Sprache zu gestalten, andere Ausdrucksformen und Lesegewohnheiten zu entwickeln - bis zum Abschied vom Text, hin zu einer Design-Kombination aus Foto, Ton, Video und Animation“ (Meier 2002: 24). Die erzählenden Websites machen von verschiedenen Ressourcen Gebrauch, indem sie sprachliche, bildliche und grafische Elemente in Form von Modulen bzw. Subflächen (vgl. Schmitz 2011) einsetzen. Je nachdem, welche Position die einzelnen Zeichen einnehmen und welche Gestalt sie haben, lassen sich ihre Funktionen bestimmen und konkretisieren, was im Folgenden am Beispiel der Online-Reportage zum Thema Germanwings-Absturz noch näher thematisiert wird.

\subsection{Online-Reportage Absturz von Flug 4 U9525 als digitales Storytelling - Korpusana- lyse}

Die hier untersuchte Geschichte des Germanwings-Absturzes ist eine große Multimedia-Reportage und setzt sich als eine Spezialform aus vielen anderen digitalen Darstellungsformen zusammen. Sturm zufolge ist es legitim, Multimedia-Reportagen als Königsdisziplin des digitalen Storytellings zu bezeichnen (vgl. Sturm 2013: 118). Die Argumente, die diese Meinung begründen, holt auch Stefan Heijnk, für den solche multimedialen Elemente wie Slideshows, Videos und Audios nicht ,als zusätzliche Klickangebote neben einem Text [stehen-MM], sondern ineinander verschachtelt, miteinander kombiniert, dramaturgisch aufeinander abgestimmt und deutlich als abgeschlossene Einheit oder gar als Website in der Website zu erkennen [sind]“ (Heijnk 2011: 286).

Die analysierte Online-Reportage konzentriert sich nicht nur auf die Katastrophe, sondern sie dient der multimodalen Dokumentation des Unglücks, der Reaktionen und Folgen des Absturzes. Daher wird sie als erzählende bzw. dokumentierende IMD klassifiziert. Die Geschichte des Germanwings-Absturzes wird in 4 Kapiteln und 44 Episoden erzählt. Das sind: Kapitel 1 Flug 
9525 (2 Episoden), Kapitel 2 Absturz und Anteilnahme (21 Episoden), Kapitel 3 Ermittlungen und Entsetzen (12 Episoden), Kapitel 4 Folgen und Verarbeitung (9 Episoden). Diese Kapitelstruktur evoziert Assoziationen mit einer literarischen Geschichte, die sich Freytag (2000) zufolge in Form eines axial-symmetrischen Dreiecks veranschaulichen lässt (Abb. 1).

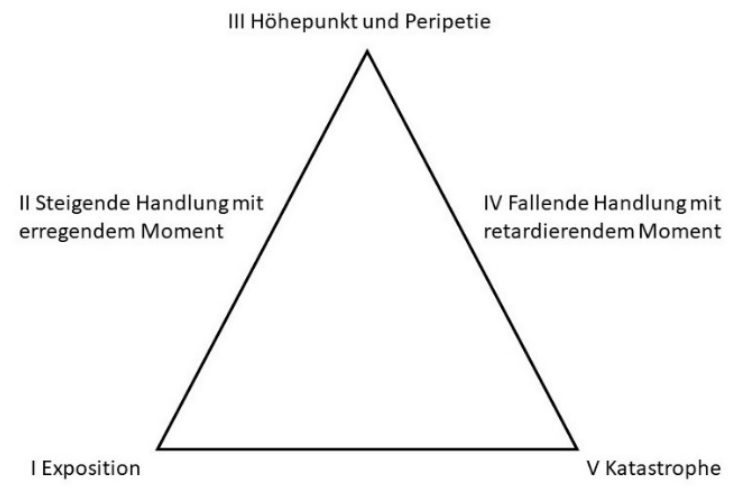

Abb. 1 Der pyramidale Bau des Dramas nach Gustav Freytag (2000: 10).

Im Folgenden wird geprüft, in wie weit die analysierte Online-Reportage dem von Freytag (2000: 10) skizzierten Schema folgt, weil diese Struktur als Grundlage für die meisten Reportagen gilt, die in der Konvention des Storytellings gestaltet werden. Die projizierte Überlappung wird bestätigen, dass es im analysierten Fall begründet ist, die Online-Reportage als Beispiel für journalistisches Storytelling zu betrachten.

\subsubsection{Kapitel 1 Flug 9525: Exposition}

Die analysierte Online-Reportage verfügt über keine extra Startseite im Sinne eines Clusters, wo man alle Struktureinheiten der Geschichte auf einer Sehfläche vor Augen haben könnte (Abb. 2). Das kann als prototypische Eigenschaft von erzählenden IMD betrachtet werden, die diesen Typus von den anderen (z. B. nachrichtenorientierten) Website-Arten unterscheidet. Wenn man eine Geschichte erzählt und an ihre Dramaturgie denkt, braucht man ihren Verlauf nicht schon am Anfang anzukündigen. Sonst sinken die innere Dynamik und Dramaturgie des Erzählens, was zur Folge haben kann, dass der Rezipient das Interesse verliert und mit der Lektüre aufhört. Im analysierten Fall gilt die erste Seite der Reportage als Einstieg in die Geschichte, der den Rezipienten zwar über die Thematik, aber nicht über die innere Gestaltung der Reportage informiert. Zu betonen ist auch das, dass die Einstiegseite gleichzeitig zum Kapitel 1 gehört, das in der ganzen Online-Reportage am kürzesten ist, aus 2 Episoden besteht und der richtigen Geschichte vorangeht, um den Blick von Usern zu fangen und sie zur weiteren Lektüre zu animieren. In dem als Exposition dienenden Kapitel 1 wird der User in die zeitlichen und räumlichen Verhältnisse eingeführt, wobei diese Einführung vor allem mittels Bilder erfolgt.

Die Einstiegseite besteht aus zwei einander überlappenden Subflächen: Als Hintergrund gilt die Bild-Subfläche, auf der rote Grablichter mit der Flugzeugnummer 9525 zu sehen sind, die symbolisch und indexalisch für den Flugzeugabsturz und die damit verbundene Trauer stehen (Abb. 2). Nicht ohne Bedeutung ist dabei ihre Farbe: Rot fällt auf und steht immer für etwas, das 
wichtig, expressiv bzw. alarmierend ist. Ein solches starkes Bild - ähnlich wie ein kurzes Video-Intro - eignet sich für die Exposition besser als der textliche Teaser, weil es den Blick des Rezipienten viel schneller fangen kann. Im Vordergrund der Einstiegseite befindet sich die Sprache-Subfläche Absturz von Flug $4 U$ 9525. Trauer, Entsetzen, Fassungslosigkeit. Sie ist stark reduziert, ,denn wo visuelles Design bereits Ordnung geschaffen hat, bleibt für Grammatik nicht mehr viel zu tun“ (Schmitz 2016: 242).

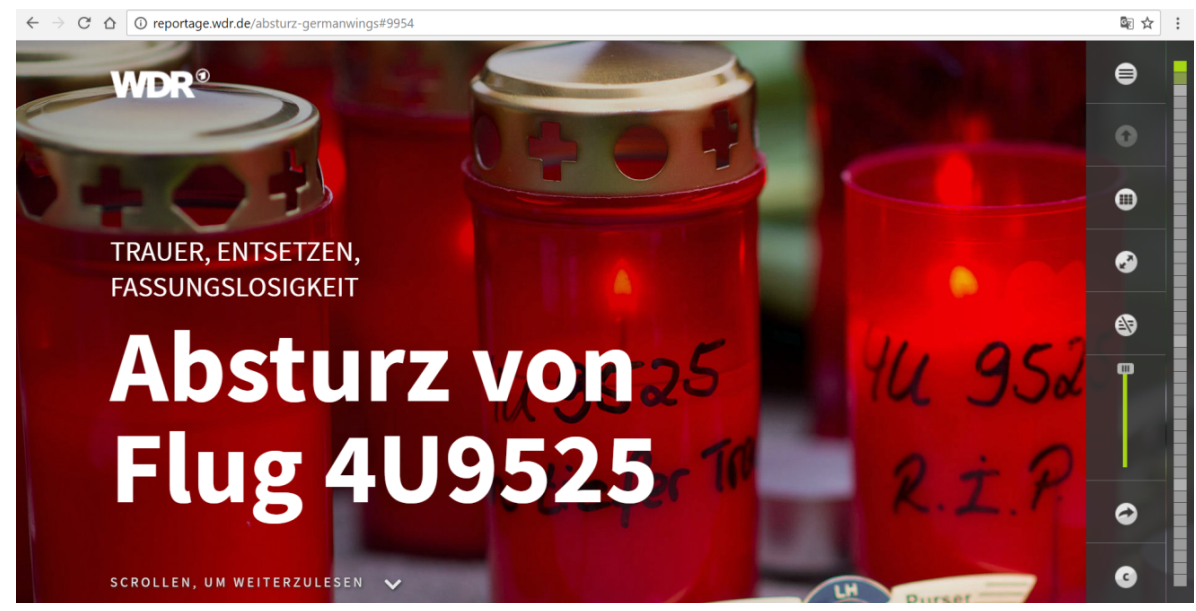

Abb. 2 Online-Reportage Absturz von Flug 4U95251

Im Falle dieser Einstiegsseite hat man mit einem Lexia-Modul zu tun, weil nicht die ganze Sehfläche ab sofort zu sehen ist und der Rezipient, der etwas mehr erfahren will, scrollen muss, um weiterzulesen. Schon an dieser Stelle, d. h. am Anfang der Geschichte, wird dem Rezipienten signalisiert, dass die IMD einer Zwei-Säulen-Struktur unterliegt, die in der ganzen Reportage konsequent beibehalten wird: Während in der linken Säule und über dem mittleren Bereich eine Abfolge von Inhalten präsentiert wird, befindet sich in der rechts anschließenden Säule das Navigationsmenü (d. h. operationale Zeichen). Identifikatorische Zeichen, darunter das Logo des WDR, zeigen sich am oberen Rand und als einheitsstiftende Elemente auf allen Unterseiten der analysierten IMD. Über die Gesamtfläche werden Ordnungszeichen verteilt, die dazu dienen, einzelne Seitenelemente zuzuordnen und abzugrenzen. An dieser Einstiegsseite ist auch gut zu erkennen, über welche Navigationsmöglichkeiten der Rezipient verfügt. Ihm stehen zwei Wege zur Wahl: Entweder liest er die Reportage wie einen linearen Text, indem er Lexia nutzt und immer weiter scrollt, oder er wählt den operationalen Weg und sucht im Navigationsmenü konkret das aus, was ihn im Moment interessiert.

\subsubsection{Kapitel 2 Absturz und Anteilnahme: Steigende Handlung mit erregendem Moment}

Dank der Exposition sollten die wichtigsten Figuren vorgestellt werden. In der analysierten Online-Reportage erfolgt das aber im Kapitel 2, das aus 21 Episoden besteht und zugleich den Großteil der Geschichte bildet. Am Anfang des Kapitels 2 lernt man die für die Handlung wesentlichen Personen kennen. Die Aufmerksamkeit des Users wird auf den Keim der Spannung gelenkt. Um dieses Ziel zu erreichen, macht man von allen technischen Möglichkeiten Ge-

\footnotetext{
${ }^{1}$ Die Quellennachweise für die Abbildungen finden sich am Ende des Beitrags.
} 
brauch, die dem digitalen Journalismus zur Verfügung stehen. So werden sowohl die Hintergründigkeit der Zeitung als auch die Bildstärke des Videos oder die Unmittelbarkeit des Rundfunks genutzt (vgl. Sturm 2013: 37; Abb. 3). Ihr paralleler Gebrauch garantiert eine interessante Entwicklung der Handlung, die man braucht, um die Aufmerksamkeit des Nutzers aufrecht zu halten.

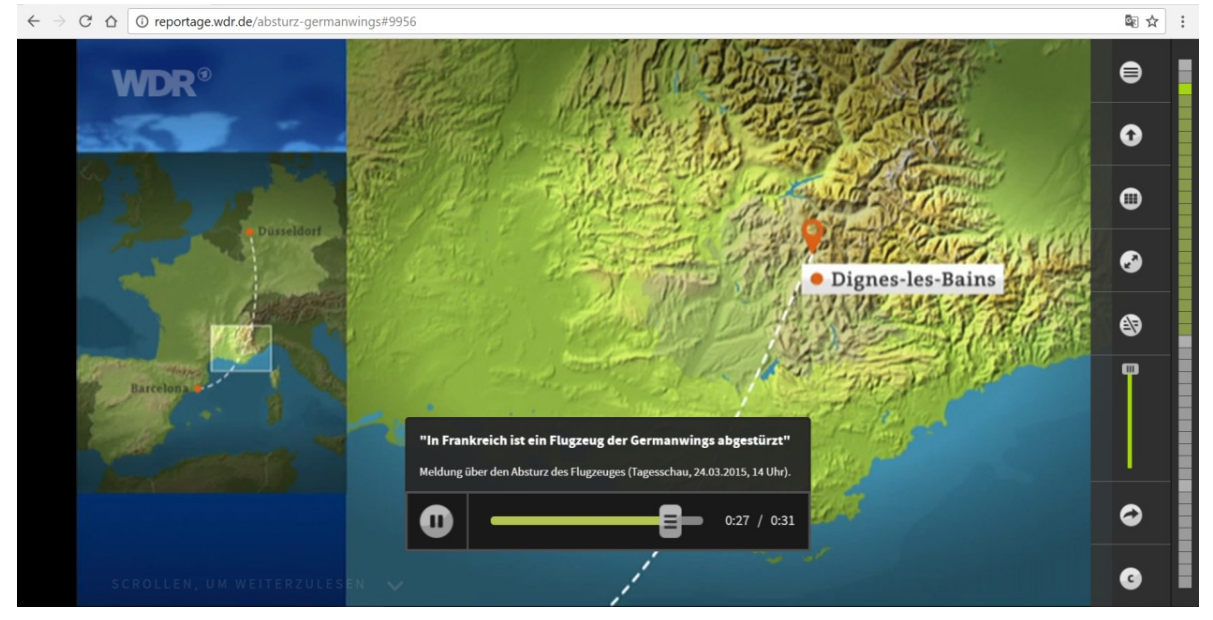

Abb. 3 Online-Reportage Absturz von Flug 4 U9525

Dabei sollte man sich danach richten, welche Elemente einer Geschichte mit Hilfe welcher Ressourcen am besten präsentiert werden können. Sprachliche Ressourcen sind zwar ideal für knappe und prägnante Informationen, aber sie wirken nicht immer so emotional wie Fotos oder Videos und verlangen oft viel mehr Konzentration und zeitlichen Aufwand. Bilder, vor allem realistische Fotos, auf denen menschliche Gesichter gut zu sehen sind, verfügen über ein besonders hohes Aktivierungspotenzial, d. h. sie richten sich viel direkter und unbewusster an die Emotionen des Rezipienten. Ein gutes Beispiel ist das Foto im Kapitel 2 (Episode 20), das Benedikt Höwedes, den aus Haltern am See kommenden deutschen Fußballspieler, kurz vor dem Spiel Deutschland-Australien darstellt (Abb. 4): Am Bord der Maschine gab es Lehrer und Schüler aus dem Joseph-König-Gymnasium in Haltern am See, die infolge der Katastrophe ums Leben gekommen sind.



Abb. 4 Online-Reportage Absturz von Flug 4 U9525 
Auch das nächste Bild (Abb. 5), auf dem die Treppe des Joseph-König-Gymnasiums zu sehen ist, die nach dem Absturz zu einer Trauerstelle geworden ist, ist sehr symbolisch.

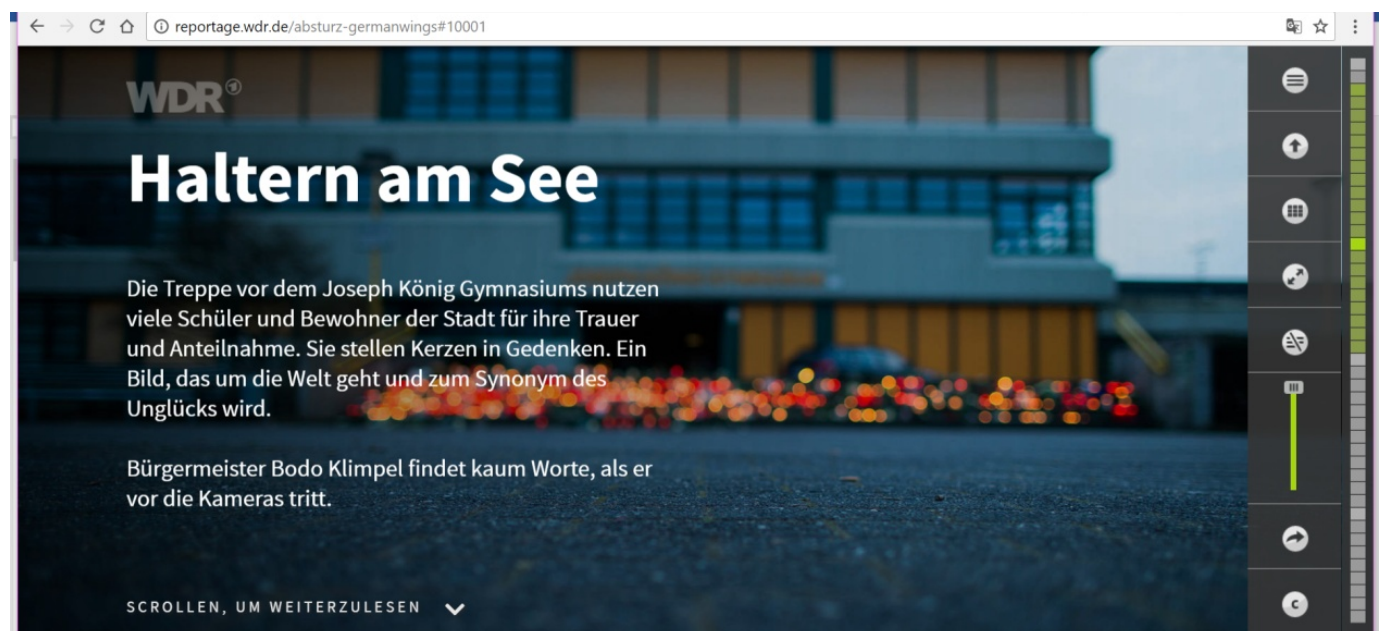

Abb. 5 Online-Reportage Absturz von Flug 4 U9525

Die beiden Fotos sind in Form von multimodalen Kommunikaten präsentiert, in denen das Bild mit einem im Vordergrund platzierten Kommentar versehen wird, der als Einstieg in die durch das Bild dominierte Sehfläche gilt. So konstruierte Kommunikate, von denen im digitalen Journalismus sehr oft Gebrauch gemacht wird, sind durch die Arbeitsteilung zwischen verschiedenen Modalitäten gekennzeichnet, die sowohl den Blick fangen als auch Informationen vermitteln. Dadurch eignen sie sich als besonders effiziente Instrumente für schnelle und komplexe Information. Sind die Fotos in einem besonderen Moment entstanden, können sie sogar eindrucksvoller als Bewegtbilder wirken. Als solche tragen sie dazu bei, dass die Handlungsfäden verknüpft werden und die Gespanntheit auf den weiteren Verlauf der Handlung steigt.

\subsubsection{Kapitel 3 Ermittlungen und Entsetzen: Höhepunkt und Peripetie \& fallende Hand- lung mit retardierendem Moment}

Der Höhepunkt der Reportage erfolgt im Kapitels 3 (Episode 24), wo man sowohl die letzten Minuten des Fluges der Germanwings-Maschine, als auch die Absturzstelle und schwierige Bergungsarbeiten beschreibt und visualisiert. Um den wahrscheinlichen Verlauf des Absturzes zu rekonstruieren, gebraucht man verschiedene Grafiken und animierte Visualisierungen, die eine hohe Glaubwürdigkeit haben (Abb. 6). „Der wesentliche Mehrwert [von Grafiken und Visualisierungen - MM] liegt darin, dass auf diese Weise abstrakte, mit Videos schwieriger oder gar nicht zu visualisierende Vorgänge lebhaft erklärt werden können“ (Sturm 2013: 122). Die in Abbildung 6 präsentierte Animation kann als Beweis dafür gelten, dass man Animationen mit anderen Formen kombinieren kann, wie z. B. mit Videos oder Fotos. Der digitale Journalismus nutzt diese Formen, weil sie auch komplizierte oder unübersichtliche Vorgänge vereinfacht darstellen und auf ihren Kern reduzieren. Deswegen ist es möglich, dank dem Gebrauch dieser Formen den Regeln der semiotischen Ökonomie zu folgen und nur das zu präsentieren, was der Situation angepasst ist. 


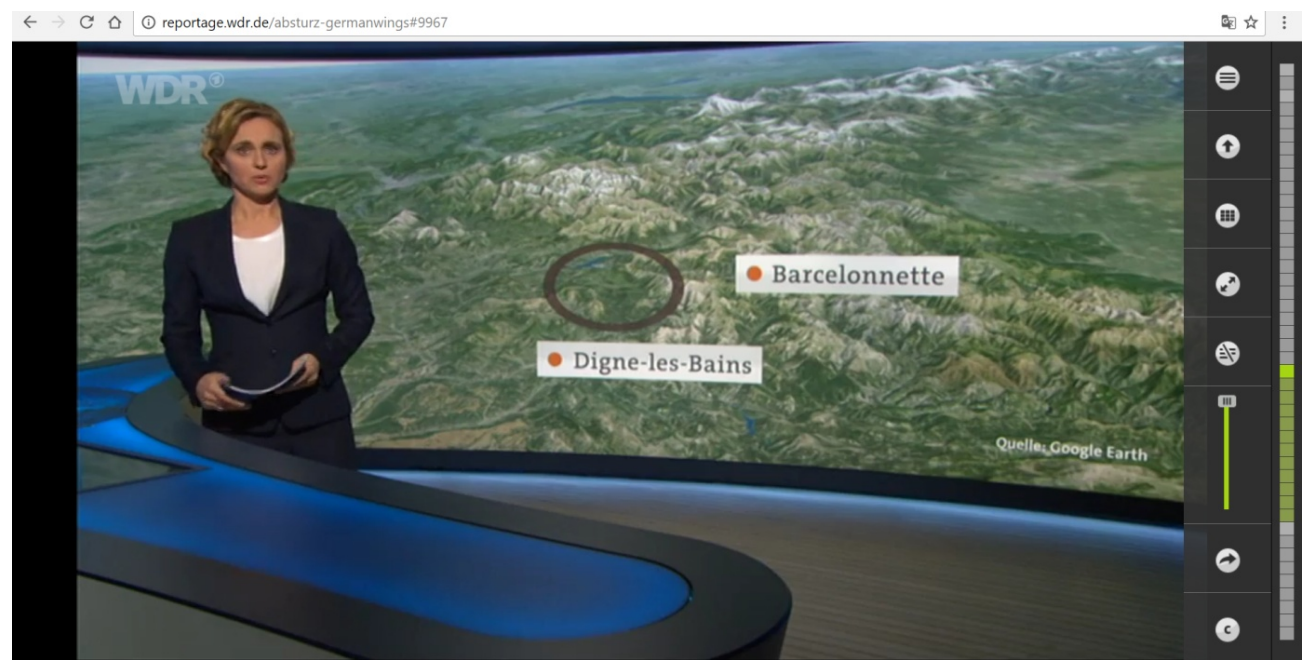

Abb. 6 Online-Reportage Absturz von Flug 4 U9525

Im Kapitel 3 (Episode 27) erfährt der User auch über die Auswertung des noch an demselben Tag an der Unglücksstelle gefundenen Stimmenrekorders (Abb. 7), was schockierende Informationen ins Licht bringt: Der Pilot versuchte, ins Cockpit zu gelangen, und der Copilot soll für den Absturz verantwortlich sein. Nach der Durchsuchung der beiden Wohnungen des Copiloten wurden weitere schockierende Informationen bekannt, nämlich dass er Krankschreibungen für den Tag des Absturzes verheimlicht hatte und mit großer Wahrscheinlichkeit das Flugzeug gezielt zum Absturz gebracht hat. Diese Informationen über die bewusste und geplante Handlung des Copiloten werden in dem Sinne zum retardierenden Moment der schon fallenden Handlung, weil sie das ganze Unglück in einem neuen Licht zeigen: Das war keine zufällige Flugzeugkatastrophe, sondern ein geplanter Absturz (Abb. 7).

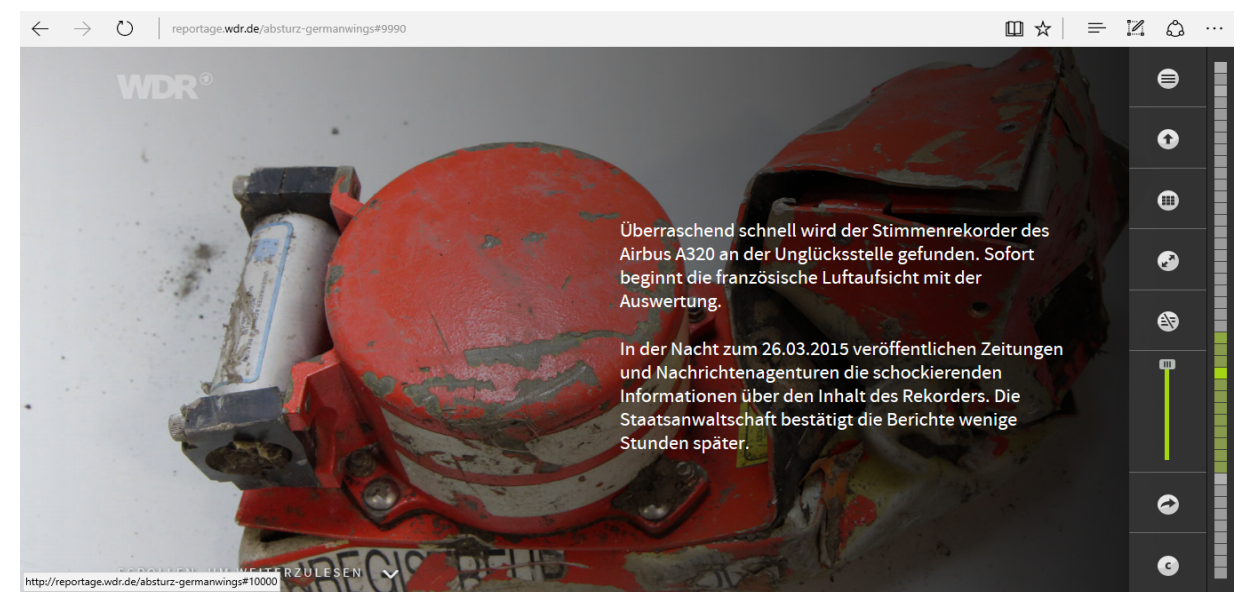

Abb. 7 Online-Reportage Absturz von Flug 4 U9525

\subsubsection{Kapitel 4 Folgen und Verarbeitung: Auflösung der Handlung}

Das letzte Kapitel 4 (Folgen und Verarbeitung) bildet den Schluss dieser Reportage und bringt die Auflösung der Handlung. Der User bekommt hier Antworten auf die Fragen, warum es zu diesem Unglück gekommen ist und ob man ihm hätte vorbeugen können. Es handelt sich einerseits um den Sicherheitsmechanismus der Cockpit-Tür, die dabei visualisiert wird (Abb. 8), und andererseits um Psychotests von Piloten, die diese und ähnliche Katastrophen verhindern könnten. Der User erfährt, dass auch die Fluggesellschaften Schlussfolgerungen ziehen: Wie in den 
USA gilt jetzt auch in Deutschland die Zwei-Personen-Regel für das Cockpit, der gemäß niemand mehr allein in der Pilotenkanzel sein darf. Einheitliche Standards bezüglich der psychischen Gesundheit von Piloten treten auch in Kraft.

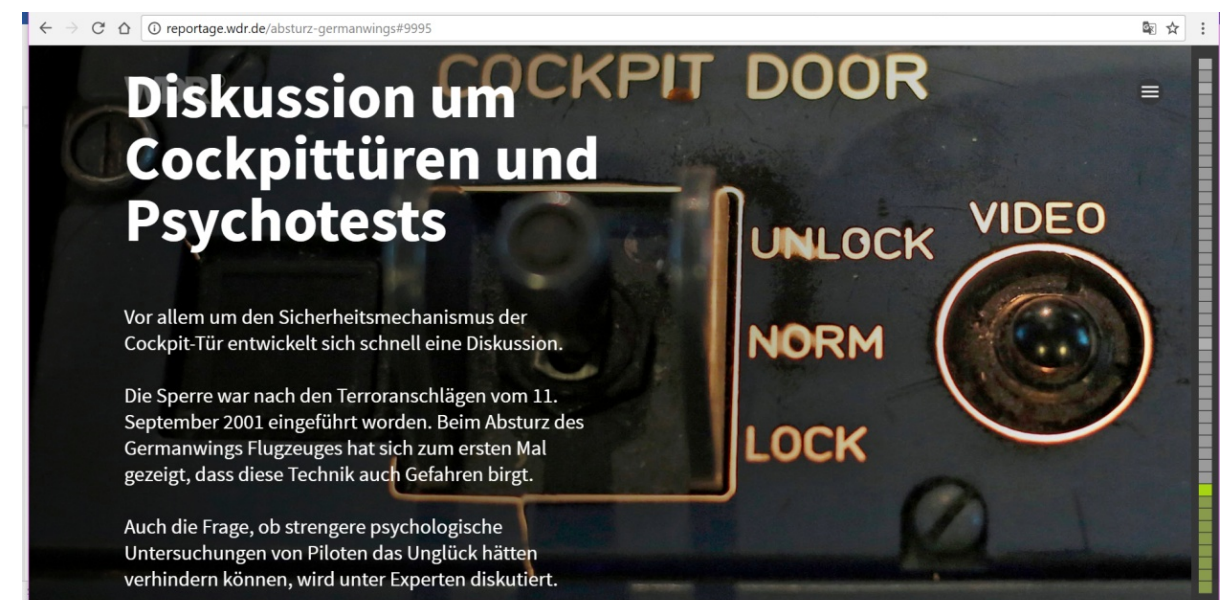

Abb. 8 Online-Reportage Absturz, von Flug 4 U9525

Um Anfang und Ende der Geschichte aufeinander zu beziehen, kann man am Ende die zu Beginn präsentierte Problematik nochmals thematisieren. In der analysierten Reportage erfolgt das u. a. mittels der Frage, ob es wieder Normalität geben wird. Diese Frage stellen sich vor allem die Familien der Opfer. Die Antwort auf diese Frage findet man z. B. in der Information, dass ein Jahr nach dem Absturz eine Schülergruppe mit dem Germanwings-Flugzeug geflogen ist. Die Geschichte macht somit einen Kreis, als ob es wirklich normal sein könnte.

Ganz am Ende gibt es eine Übersicht, dank der der User die Möglichkeit hat, die Struktur der ganzen Online-Reportage nochmals zu überblicken (Abb. 9). So erfährt er, wie diese Geschichte innerlich gestaltet wird, was besonders dann von Bedeutung ist, wenn er etwas nochmals sehen, hören bzw. lesen möchte. Dank dieser Übersicht lässt sich feststellen, dass die ganze Geschichte modular erzählt wird und dass den so genannten Mikrotexten, wie z. B. Kurzteasern, die zu weiteren Textpassagen führen und dazu beitragen, dass der Leser der Geschichte folgt und die IMD nicht verlässt, besondere Bedeutung zukommt.

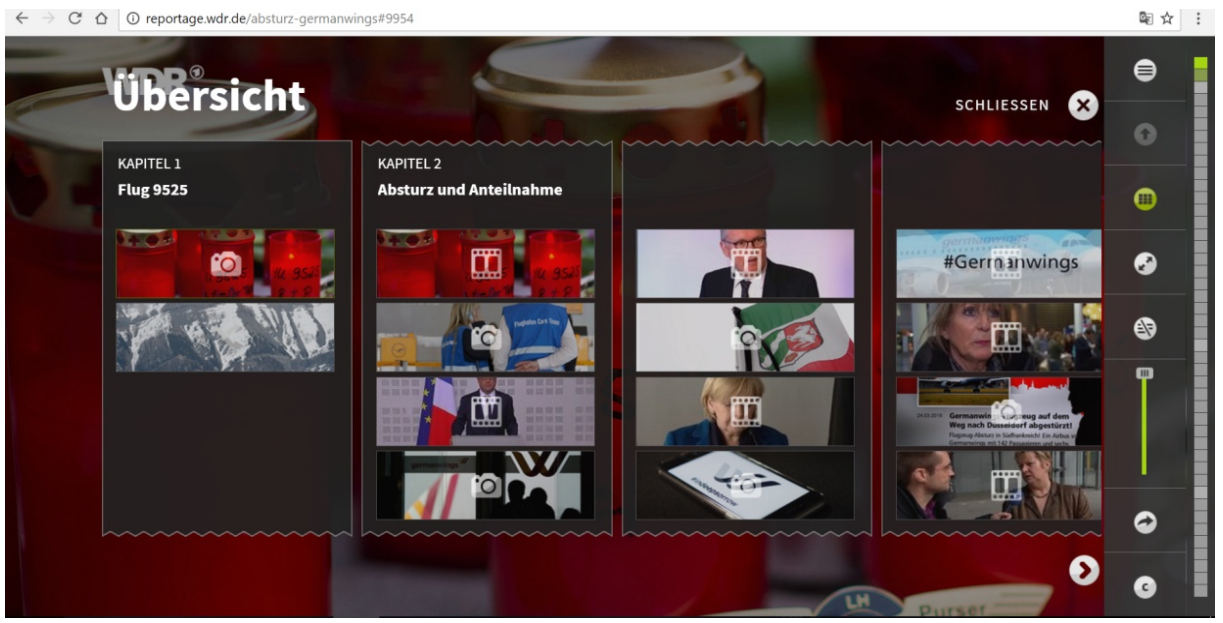

Abb. 9 Online-Reportage Absturz von Flug 4 U9525 
Diese Übersicht veranschaulicht auch, dass mittels multimodaler Teaser dem Rezipienten eine ganze Sehfläche zur Verfügung gestellt wird und er sie nonlinear rezipieren kann. Es hängt ausschließlich von seiner Entscheidung ab, welchen Weg er gehen möchte, ob er die ganzen Unterkapitel nochmals analysiert oder nur die einzelnen Sequenzen herausfiltert. Die Platzierung dieser Übersicht ganz am Ende der Online-Reportage unterscheidet diese erzählende IMD von den anderen, schon oben angesprochenen Typen: Während eine nachrichtenorientierte Website eine solche Übersicht zum Einstieg macht, nutzt man diese Übersicht im Falle einer erzählenden Website erst als Zusammenfassung dessen, was in den einzelnen Kapiteln und Episoden präsentiert und erzählt wurde. Somit wird die Funktion der Online-Reportage und der dabei gebrauchten erzählenden bzw. dokumentierenden IMD konkretisiert und verdeutlicht.

\subsection{Kohärenz- und kohäsionsstiftende Elemente von IMD}

Die im Rahmen des digitalen Storytellings präsentierten Geschichten sind durch ihre multimodale Realisierung gekennzeichnet, die verschiedene Ebenen betrifft und viele kohäsion- und kohärenzstiftende Elemente enthält (vgl. Meier 2016, Stöckl 2016a):

a. Erstens wird dank diverser Mittel die interne Gliederung der Geschichte signalisiert. Die einzelnen Module, die von sprachlichen und/oder bildlichen Ressourcen Gebrauch machen, tragen dazu bei, dass die ganze Geschichte strukturiert wird. Ihre Binnenstruktur wird dem Rezipienten im Prozess der Wahrnehmung bewusst und ist bei der Einordnung von Handlungen und Themen behilflich. Mittels Linien, Rahmen und Texturwechsel werden auch die Grenzen der Geschichte gegenüber benachbarten Kommunikationsformen signalisiert. Im digitalen Journalismus wird also der multimodalen Modularisierung die Rolle im Prozess der internen und externen Strukturierung zugeschrieben.

b. Zweitens erleichtert die multimodale Modularisierung auch Anordnungen von funktionalen Handlungsabschnitten. Die Stärken und Schwächen der einzelnen Modalitäten werden berücksichtigt, um dem Rezipienten die beste Art und Weise der Darstellung von Inhalten zu garantieren.

c. Drittens ist die Rolle der multimodalen Modularisierung auch in dem Sinne von Bedeutung, dass sie zu einer besseren Darstellung von Teilthemen beitragen kann. Wichtig ist sowohl das, welche Teilthemen die einzelnen Modalitäten zum Gesamttext beitragen, als auch das, welche Kombinationen welcher Ressourcen zur Darstellung von einzelnen Teilthemen dienen.

d. Viertens ist zu fragen, in welchen Kombinationen die einzelnen Modalitäten am ergonomischsten sind, d. h. z. B. welche Verflechtungen als besonders kohäsiv bzw. kohärent klassifiziert werden können. Dabei geht es auch darum, festzustellen, welche Funktionen die verwendeten Modalitäten in wechselseitiger Bezogenheit füreinander übernehmen können.

Wie schon unter 3.2 dargelegt wurde, zeigt die im vorliegenden Beitrag analysierte OnlineReportage ihre eigene innere Strukturierung in Kapiteln und Episoden und ist auch durch Modularisierung auf einzelnen Sehflächen geprägt. Einige solcher Module wie z. B. das rechts platzierte Menü sind stets präsent und dienen als kohäsionsstiftende Elemente der IMD-Struktur. Mittels solcher Elemente wird dem User signalisiert, dass er mit einer einheitlichen Botschaft zu tun hat und dass alle Informationen, die auf den einzelnen Seiten präsentiert werden, einen Gesamttext bilden. Auch der Einsatz anderer typografischer Mittel, wie z. B. Schriftstil 
und -größe, Linien/Rahmen sowie Form und Farbe, trägt dazu bei, dass die einzelnen Teile der IMD als eine semantische, funktionale und strukturelle Einheit wahrgenommen und interpretiert werden. Aufgrund der einheitlichen Positionierung entstehen wiedererkennbare Textformen, die dem User den Umgang mit der IMD wesentlich erleichtern. Diese textgraphische Konturierung bildet die Basis für die Etablierung von Handlungs- und Themenstruktur. Während mit der Überschrift der Inhalt angekündigt wird, berichten der Textkörper und das Video die Details. Wird von einer Infografik Gebrauch gemacht, so werden im Textkörper Fragen gestellt, die dann mittels Bilder bzw. Diagramme beantwortet werden. „Gemeinsam ist den multimodalen Textsorten also, dass sie die einzelnen Handlungen (stages) klar auf die Modalitäten verteilen und dass Sprache dabei eine zentrale Stellung einnimmt, wenn ihr Status auch anteilsmäßig verschieden ist" (Stöckl 2016a: 26).

In allen Teilen der Geschichte werden die Zeichenmodalitäten so miteinander verknüpft, dass eine spezifische Form von Kohäsion und Kohärenz unter ihnen entsteht. Diese Verknüpfungen basieren darauf, dass sich die einzelnen Modalitäten gegenseitig ergänzen und zur Bildung des Gesamttextes wesentlich beitragen. Schubert zufolge ist es begründet, in diesem Kontext zwischen lexikalischer und grammatischer Kohäsion zu unterscheiden (vgl. Schubert 2012: 4648). Als lexikalische Kohäsion sind u. a. Wiederholungen und Paraphrasen zu klassifizieren, die im analysierten Fall vor allem aus den Wortfeldern Flugzeugkatastrophe und Trauer kommen. Als Beweis dafür, dass man im Falle des digitalen Journalismus auch mit so genannter grammatischer Kohäsion zu tun haben kann, gelten Elemente der Konnektivität, die auf andere Stellen im Text verweisen. Dazu gehören z. B. Demonstrativ- und Personalpronomina, Ellipsen oder Konjunktionen. Schubert weist aber auch darauf hin, dass Elemente der Textgestaltung wie Linien, Balken oder Pfeile als Faktoren der grammatischen Kohäsion betrachtet werden können (vgl. ebd.). Im Zusammenhang mit der so realisierten Kohäsion steht auch die Kohärenz, die vor allem dann gestiftet wird, wenn der Rezipient anhand der Mittel der lexikalischen Kohäsion und im Prozess von deren Interpretation logisch-semantische Relationen zwischen einzelnen Propositionen feststellt. Schon die Einstiegsseite der analysierten Online-Reportage kann als Beispiel dafür gelten, wie man logisch-semantische Relationen zwischen den einzelnen Modalitäten herstellen kann (Abb. 2). Der lexikalischen Ebene liegt die Phrase Absturz von Flug 4U9525 zugrunde, die mikrotypografisch hervorgehoben wird. Auf dem roten Hintergrund sind fett markierte, weiße und große Buchstaben gut zu sehen. Über ihnen findet der Rezipient drei Nomen: Trauer, Entsetzen, Fassungslosigkeit, die als Reaktion auf die Katastrophe zu verstehen sind und lexikalisch zum Wortfeld Absturz gehören. Die Abbildung, in der rote Grablichter zu sehen sind, dient somit als Hintergrundinformation zu dem im Titel thematisierten Absturz: Mit Hilfe von den Grablichtern wird gezeigt, dass es Todesopfer gibt. Dieses Bildmotiv sorgt somit ganz stark für multimodale Kohärenz und wird zum Hauptakteur in dem der Einstiegsseite zugrundeliegenden Skript Absturz.

Die lexikalische Kohäsion wird auch dann sichtbar, wenn man die benachbarten Seiten der Online-Reportage analysiert (Abb. 10 und 11). Das in Abbildung 10 gebrauchte Bild, auf dem ein Smartphone zu sehen ist, dient der Visualisierung dessen, was in der Sprache-Subfläche versprachlicht wird. Diese Sehfläche (Kapitel 2, Episode 10) thematisiert das Mitgefühl mit den Opfern und ihren Angehörigen, was seine Widerspiegelung in Form dieses Bildes findet: Es ist dunkel, eigentlich fast schwarz-weiß: Im europäischen Kulturkreis gilt die schwarze Farbe als 
Ausdruck der Trauer. Man visualisiert somit die in den sozialen Netzwerken registrierten Reaktionen auf den Absturz.

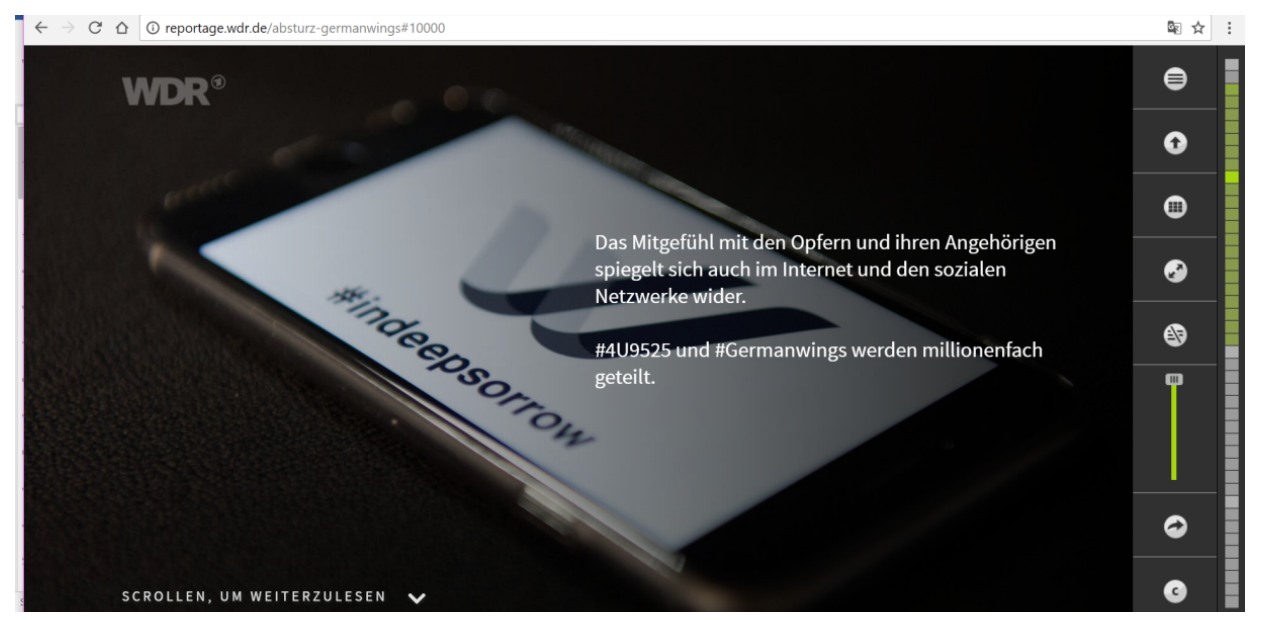

Abb. 10 Online-Reportage Absturz von Flug 4 U9525

Einige Beispiele für diese Reaktionen sind auch auf der nächsten Sehfläche (Kapitel 2, Episode 11, Abb. 11) zu sehen. Außer dem Hashtag \#Germanwings, das eine direkte Vernetzung der beiden Sehflächen sichert, gibt es hier erste emotionale Reaktionen von schockierten Netzbenutzern. Dazu kommen noch Videosequenzen, in denen andere Personen, z. B. Passagiere am Flughafen, interviewt werden.

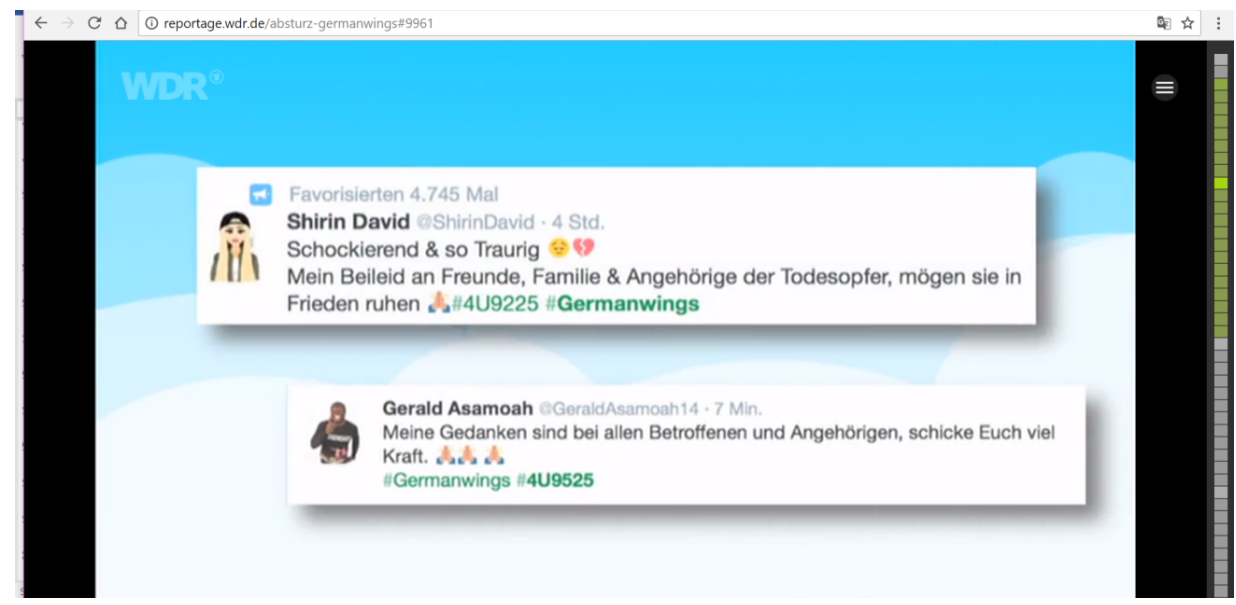

Abb. 11 Online-Reportage Absturz von Flug 4 U9525

\section{$4 \quad$ Schluss}

Im digitalen Storytelling spielt die Multimodalität eine immer wichtigere Rolle. Multimodale Elemente machen den Rezipienten neugierig und fangen seinen Blick, wodurch die Effektivität des Geschichtenerzählens gesteigert wird. Sie reichen aber nicht aus, wenn die Geschichte nicht spannend konstruiert wird und nur gut verpackt ist. Nur diejenigen Geschichten, die gut erzählt werden, sind in der Lage, den Rezipienten direkt anzusprechen und ihn emotional zu engagieren. Im digitalen Storytelling können Rezipienten sowohl visuell als auch auditiv angesprochen werden. Die visuelle Animierung erfolgt mittels verbaler und bildlicher Ressourcen, wobei die letzteren sowohl statische bzw. bewegte Bilder als auch 
Diagramme und Infografiken umfassen. Auditive Animierung erfolgt meistens in Form von Videos. Jede Art der Informationsvermittlung verfügt dabei über ihre Stärken und Schwächen. Das digitale Storytelling ist deswegen von Vorteil, weil es die Stärken einer Modalität nutzt und die Schwächen einer anderen ausgleicht, indem es zu Mitteln greift, die sich für eine konkrete Geschichte und ein konkretes Teil-Thema besonders gut eignen. Nur eine sinnvolle Verknüpfung unterschiedlicher Zeichenmodalitäten führt zur Herstellung von intermodaler Kohäsion bzw. Kohärenz.

Die analysierte Online-Reportage, die als eine erzählende IMD zu klassifizieren ist, zeigt, dass es legitim ist, journalistisches Storytelling für ein sehr flexibles Mittel der Beschreibung und Erklärung von Wirklichkeit zu halten. Sie zeigt, dass multimodales Storytelling dem Journalismus attraktive Formen der Darstellung und Verbreitung von Informationen bieten kann und wie es neue technische Möglichkeiten für moderne Formen von Narrativität nutzt, um öffentlich relevante Themen mit neuen Ressourcen dramaturgisch attraktiv aufzubereiten und zu dokumentieren. Das digitale Storytelling verändert fundamental die Art und Weise, wie eine Geschichte aufgenommen und verbreitet wird: Der gut durchdachte Gebrauch von sprachlichen und bildlichen, in Module bzw. Subflächen aufgeteilten Ressourcen ermöglicht die Optimierung des dynamischen Geschichtenerzählens und trägt zur Entstehung der visuellen Narration bei.

\section{Literaturverzeichnis}

Becker, Alexander (2010): „Man kann nie sagen, wir haben es geschafft“. https://meedia.de/2010/10/26/man-kann-nie-sagen-wir-haben-es-geschafft. [08.11.2018]

Bucher, Hans-Jürgen (2012): „Grundlagen einer interaktionalen Rezeptionsforschung: Einführung und Forschungsüberblick“. In: Bucher, Hans-Jürgen/Schumacher, Peter (eds.): Interaktionale Rezeptionsforschung. Theorie und Methode der Blickaufzeichnung in der Medienforschung. Wiesbaden, Springer: 17-50.

Bucher, Hans-Jürgen/Schumacher, Peter (2012): „Einleitung: Interaktionale Rezeptionsforschung“. In: Bucher, Hans-Jürgen/Schumacher, Peter (eds.): Interaktionale Rezeptionsforschung. Theorie und Methode der Blickaufzeichnung in der Medienforschung. Wiesbaden, Springer: 9-14.

Chiew, Arthur Kok Kum (2004): „Multisemiotic mediation in hypertext“. In: O’Halloran, Kay L. (ed.): Multimodal Discourse Analysis. London, New York, Continuum: 131-162.

Freytag, Gustav (2000): Die Technik des Dramas. Ditzingen: Reclam

Handler, Peter (2006): „Zur Narrativik des „,digital storytelling“. Textkonstitution in sequenziell angelegten Web-Formaten“. Germanistische Linguistik 186: 209-234.

Heijnk, Stefan (2011): Texten fürs Web. Planen, schreiben, multimedial erzählen. Das Handbuch für Online-Journalisten. 2. überarbeitete und erweiterte Auflage. Heidelberg: dpunkt Verlag.

Hess-Lüttich, Ernest W. B. (1997): „Text, Intertext, Hypertext - Zur Texttheorie der Hypertextualität“". In: Klein, Josef/Fix, Ulla (eds.): Textbeziehungen. Linguistische und literaturwissenschaftliche Beiträge zur Intertextualität. Tübingen, Stauffenburg Verlag: 125-148.

Jewitt, Carey (2014): "What next for multimodality?" In: Jewitt, Carey (ed.): The Routledge handbook of multimodal analysis. New York: Routledge: 450-455. 
Lilienthal, Volker (2011): „Qualität unter Druck - Journalismus im Internetzeitalter“. In: Schwanebeck, Axel/Schröder, Michael (eds.): Qualität unter Druck: Journalismus im Internet-Zeitalter. Baden-Baden, Nomos: 49-59.

Meier, Klaus (20023): Internet-Journalismus. Konstanz: UVK.

Meier, Stefan (2016): „Websites als multimodale digitale Texte“. In: Klug, Nina-Maria/Stöckl, Hartmut (eds.): Handbuch Sprache im multimodalen Kontext. Berlin/Boston, de Gruyter: 410-436.

Opiłowski, Roman (2015): Der multimodale Text aus kontrastiver Sicht. Textdesign und Sprache-Bild-Beziehung in deutschen und polnischen Pressetexten. Wrocław - Dresden: ATUT/Neisse.

Perrin, Daniel (2010): „Wie sich Formate verändern. Die Mustervariation der Geschichte in Fernsehnachrichten“. In: Bucher, Hans-Jürgen/Gloning, Thomas/Lehnen, Katrin (eds.): Neue Medien - neue Formate. Ausdifferenzierung und Konvergenz in der Medienkommunikation. Frankfurt, Campus: 145-165.

Pethes, Nicolas (2008): Kulturwissenschaftliche Gedächtnistheorien zur Einführung. Hamburg: Junius.

Schmitz, Ulrich (2001): „Stets heikle Kohärenz in Text-Bild-Gefügen. Sinnsuche aufs Papier und Sinnkonstruktion am Computer". In: Hess-Lüttich, Ernest W.B. (ed.): Medien, Texte und Maschinen. Wiesbaden, VS Verlag für Sozialwissenschaften: 141-165.

Schmitz, Ulrich (2011): „Sehflächenforschung. Eine Einführung“. In: Diekmannshenke, Hajo/Klemm, Michael/Stöckl, Hartmut (eds.): Bildlinguistik. Theorien - Methoden - Fallbeispiele. Berlin, Erich Schmidt: 23-42.

Schmitz, Ulrich (2016): „Sprachwandel im Augenblick - Kleinteilige Texte auf Sehflächen“. In: Kwekkeboom, Sarah/Waldenberger, Sandra (Hg.): PerspektivWechsel oder: die Wiederentdeckung der Philologie. Bd. 1, Sprachdaten und Grundlagenforschung in der Historischen Linguistik. Berlin, Erich Schmidt: 233-247.

Schröder, Thomas (2010): „Die Bilder-Zeitung: Wie ein Textmedium zu einem Medium der visuellen Kommunikation wird“. In: Bucher, Hans-Jürgen/Gloning, Thomas/Lehnen, Katrin (eds.): Neue Medien - neue Formate. Ausdifferenzierung und Konvergenz in der Medienkommunikation. Frankfurt, Campus: 169-188.

Schubert, Christoph (2012²): Englische Textlinguistik. Eine Einführung. Berlin: Erich Schmidt. Schumacher, Peter (2012): „Zur Usability multimodaler Darstellungsformen im Onlinejournalismus“. In: Bucher, Hans-Jürgen/Schumacher, Peter (eds.): Interaktionale Rezeptionsforschung. Theorie und Methode der Blickaufzeichnung in der Medienforschung. Wiesbaden, Springer: 311-323.

Stöckl, Hartmut (2016a): „Multimodalität - Semiotische und textlinguistische Grundlagen“. In: Klug, Nina-Maria/Stöckl, Hartmut (eds.): Handbuch Sprache im multimodalen Kontext. Berlin/Boston, de Gruyter: 3-35.

Stöckl, Hartmut (2016b): „Multimodalität im Zeitalter des Social Web: Eine forschungsmethodische Skizze“. In: Beachler, Coline/Eckkrammer, Eva Martha/Müller-Lancé, Johannes/Thaler, Verena (eds.): Medienlinguistik 3.0 - Formen und Wirkungen von Textsorten im Zeitalter des Social Web. Berlin, Frank \& Timme: 21-30.

Sturm, Simon (2013): Digitales Storytelling. Eine Einführung in neue Formen des Qualitätsjournalismus. Wiesbaden: Springer. 
Żebrowska, Ewa (2013): Text - Bild - Hypertext. Frankfurt am Main, Berlin, New York: Lang.

\section{Abbildungsnachweise:}

Abb. 2: http://reportage.wdr.de/absturz-germanwings\#9954 [13.07.2017].

Abb. 3: http://reportage.wdr.de/absturz-germanwings\#9956 [13.07.2017].

Abb. 4: http://reportage.wdr.de/absturz-germanwings\#9986 [13.07.2017].

Abb. 5: http://reportage.wdr.de/absturz-germanwings\#10001 [13.07.2017].

Abb. 6: http://reportage.wdr.de/absturz-germanwings\#9967 [13.07.2017].

Abb. 7: http://reportage.wdr.de/absturz-germanwings\#9993 [13.07.2017].

Abb. 8: http://reportage.wdr.de/absturz-germanwings\#9995 [13.07.2017].

Abb. 9: http://reportage.wdr.de/absturz-germanwings\#10021 [13.07.2017].

Abb. 10: http://reportage.wdr.de/absturz-germanwings\#10000 [13.07.2017].

Abb. 11: http://reportage.wdr.de/absturz-germanwings\#9961 [13.07.2017]. 